\title{
Formulation design facilitates magnetic nanoparticle delivery to diseased cells and tissues
}

\begin{abstract}
Magnetic nanoparticles (MNPs) accumulate at disease sites with the aid of magnetic fields; biodegradable MNPs can be designed to facilitate drug delivery, influence disease diagnostics, facilitate tissue regeneration and permit protein purification. Because of their limited toxicity, MNPs are widely used in theranostics, simultaneously facilitating diagnostics and therapeutics. To realize therapeutic end points, iron oxide nanoparticle cores $(5-30 \mathrm{~nm})$ are encapsulated in a biocompatible polymer shell with drug cargos. Although limited, the toxic potential of MNPs parallels magnetite composition, along with shape, size and surface chemistry. Clearance is hastened by the reticuloendothelial system. To surmount translational barriers, the crystal structure, particle surface and magnetic properties of MNPs need to be optimized. With this in mind, we provide a comprehensive evaluation of advancements in MNP synthesis, functionalization and design, with an eye towards bench-to-bedside translation.
\end{abstract}

Keywords: biocompatible polymer coat $\bullet$ drug delivery $\bullet$ magnetic nanoparticle

- MRI • nanotoxicology • protein purification • superparamagnetic properties $\bullet$ surface

functionalization $\bullet$ targeting ligands $\bullet$ theranostics $\bullet$ therapeutic index

Nanomedicines are developed to improve disease diagnosis and therapeutic indices for a range of injuries, infectious diseases, genetic and immune disorders and cancers [1]. One way to broaden the impact of nanomedicine is through theranostics, a means by which magnetic nanoparticles (MNPs) serve to facilitate both therapeutic and diagnostic end points [2]. MNPs were developed for labeling, isolation and purification of cells, proteins, carbohydrates, antibiotics, biopolymers and other biomolecules. Additional applications include tumor hyperthermia, tissue engineering, drug delivery and disease diagnostics through MRI [3-8]. MNPs are 15-30 $\mathrm{nm}$ in size and possess intrinsic superparamagnetic properties, enabling their transport to, and retention at, specific body sites aided by external magnetic fields [9]. Therapeutic MNPs contain iron oxide cores of $<15 \mathrm{~nm}$, a biocompatible polymer coat, and targeting ligands and use MRI for detection and enablement of disease site delivery [10-13] (Figure 1). Similar approaches have been described for the design of nanotherapeutics for the treatment of HIV infection [14].

MNPs need to be biocompatible, elicit limited toxicity and effectively accumulate at disease sites to be effective [15]. The ironcontaining particles also need to dissolve and release their contents for clearance by the body's reticuloendothelial system (RES) [16,17]. MNP toxicity is linked to composition, shape, size and surface chemistry. Manufacturing parameters can limit inherent toxicities [18], enabling a broad use in biomedical applications [19]. The US FDA has approved MNPs as MRI contrast agents [20]. When drugs are encased in the nanoparticles, MNPs may evaluate drug pharmacokinetics and biodistribution. Future therapeutic values of MNPs rest in drug targeting, hyperthermia-induced tumor destruction and in rapid disease diagnoses [18]. It is noteworthy that materials such as cobalt, which show significant magnetic responses, are not suited for clinical use based on their toxicity profiles [19].
Dhirender Singh ${ }^{1,2}$, JoEllyn M McMillan ${ }^{* 2}$, Xin-Ming Liu ${ }^{1,2}$, Hemant $M$ Vishwasrao ${ }^{3}$, Alexander V Kabanov $^{3}$, Marina SokolskyPapkov $^{3}$ \& Howard E Gendelman ${ }^{1,2}$

'Department of Pharmaceutical Sciences, University of Nebraska Medical Center, Omaha, NE, USA

${ }^{2}$ Department of Pharmacology

\& Experimental Neuroscience, University of Nebraska Medical Center, Omaha, NE, USA

${ }^{3}$ Center for Nanotechnology in Drug Delivery, UNC Eshelman School of

Pharmacy, University of North Carolina at Chapel Hill, Chapel Hill, NC, USA *Author for correspondence: Tel.: +1 4025593074 Fax: +1 4025597495 jmmcmillan@unmc.edu 


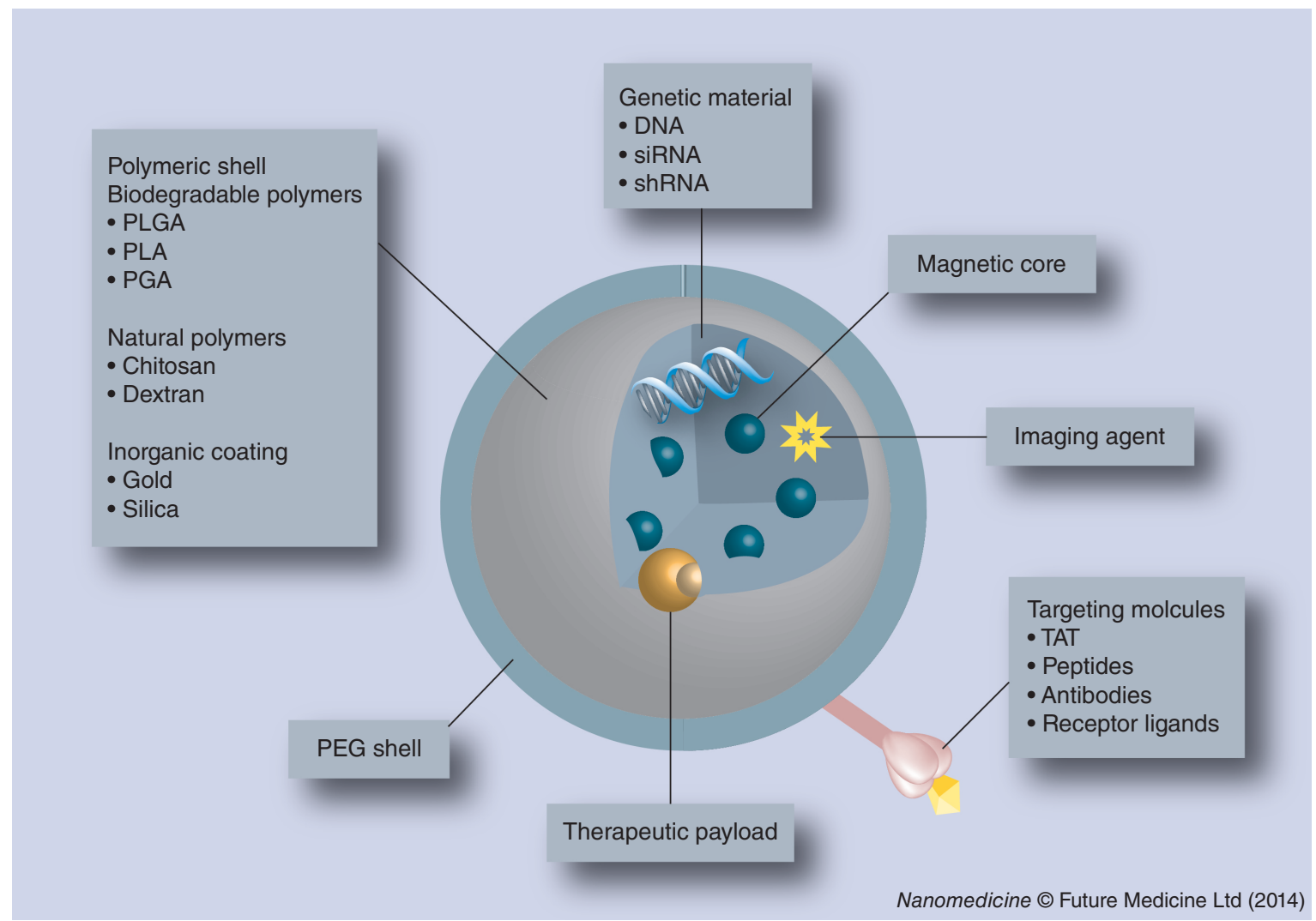

Figure 1. Basic features of multifunctional composite magnetic nanoparticles. Magnetic nanoparticles are capable of targeted delivery of a therapeutic cargo (drug, genetic material and/or imaging agent) under the influence of an external magnetic field. The components of multifunctional composite magnetic nanoparticles include a biocompatible polymeric shell (biodegradable, inorganic or natural polymers) that encapsulates the magnetic core along with the therapeutic payload.

PEG: Polyethylene glycol; PGA: Polyglycolide; PLA: Polylactide; PLGA: Poly(lactide-co-glycolide).

In this article, we discuss the various methods used to synthesize MNPs for targeted applications in diagnostics and therapeutics. Specific physicochemical and physical parameters that are important for the design of effective targeted delivery of MNPs are also reviewed.

\section{Formulation developments \& product synthesis}

Typically, MNPs contain a coated magnetic core of either magnetite $\left(\mathrm{Fe}_{3} \mathrm{O}_{4}\right)$ or its oxidized forms, maghemite and hematite [21]. Synthesis of MNPs is controlled by concentration of reactants, mixing rate, oxidation rate and temperature and for aqueous route by $\mathrm{pH}$ [22]. The morphology of MNPs revolves around their nucleation, aggregation and impurities [23]. Different models of particle nucleation and growth have been reported. LaMer and co-workers developed a model for MNP development [24,25], which is as follows: first, monomer concentration gradually increases until it reaches supersaturation; second, supersaturated solutions of monomers undergo nucleation; third, nucleation ensues resulting in the formation of large numbers of nuclei termed 'bursts'. A pure solution results in homogeneous nucleation, whereas impurities, such as dust, initiate heterogeneous nucleation. Nucleation continues until monomer concentration drops below a critical concentration; fourth, particles continue to grow through absorption of additional monomers on their surfaces. As monomer adsorption is not homogeneous and depends on the surface free energy, nanoparticulate matter of very high surface free energy results in particles with broad size distribution [26]. To control nanoparticle growth, surfactant molecules, which consist of a hydrocarbon chain attached to a functional end group, are used. Surfactants bind to particles though the functional group, thus limiting particle surface reactivity and further particle growth. In addition, hydrocarbon chains form a hydrophobic shell around the magnetite/maghemite core, providing stability against oxidation to formed nanoparticles [27-29]. Over the past 30 years, numerous synthetic approaches were established to obtain reliable MNPs with optimum size, shape, surface charge and colloidal stability. These include co-precipitation, hydrothermal and microemulsion syntheses and thermal 
decomposition. Each of these 'bottom-up' nanoparticle formation techniques provides more control over particle size, size distribution and phase purity than mechanical grinding. These synthetic methods are summarized and compared in Table 1.

\section{Co-precipitation}

Co-precipitation is the most common technique for MNP manufacture. Hydrophilic magnetite/maghemite nanoparticles are produced by addition of alkali to an aqueous salt solution of $\mathrm{Fe}^{2+/} \mathrm{Fe}^{3+}$ at room temperature under an inert atmosphere [30]. The resulting particles require annealing, which precludes agglomeration. Co-precipitation performed at higher temperatures $\left(50-100^{\circ} \mathrm{C}\right)$ leads to condensation of hydroxides to crystalline metal oxides [31]. At first magnetite particles are formed and, if subjected to controlled oxidation, are transformed into stable maghemite particles. Controlled transformation of magnetite to maghemite can be achieved by dispersing the magnetite particles in an acidic medium followed by addition of $\mathrm{Fe}^{3+}$ nitrate or nitric acid. Maghemite particles are more stable than magnetite over long time periods as they are not sensitive to oxidation [31,32]. Co-precipitation is a reproducible and high yield synthesis method [30]; however, synthesis of particles with narrow size distribution still remains challenging [33]. Size, shape and particle composition is controlled by $\mathrm{Fe}^{2+} / \mathrm{Fe}^{3}$ ratios and $\mathrm{pH}$ and ionic strength; the latter two also impact chemical composition and charge at the particle surface. Particle size and size distributions decrease at high $\mathrm{pH}$ and ionic strength [33,34]. Passing nitrogen gas through the reaction can decrease particle size and provide protection against oxidation [35]. Monodispersed nanoparticles can also be synthesized by decreasing the nucleation period. Another method to control the size and shape of the magnetic particles is 'in situ formation', wherein co-precipitation is conducted in a polymer matrix with cavities of a preset size and shape, which serve as a template for particle formation, thus reducing particle polydispersity [36].

\section{Hydrothermal synthesis}

Hydrothermal synthesis is conducted at a temperature and pressure above $200^{\circ} \mathrm{C}$ and 2000 psi, respectively. Hydrothermal MNP synthesis proceeds by hydrolysis and oxidation of ferrous salt or by neutralization of mixed metal hydroxides [37] and promotes rapid nucleation and growth of smaller high quality crystals $[25,38]$. When metal salts are dissolved under ambient conditions, hydrothermal synthesis can proceed at supercritical fluid temperatures [39]. Hydrothermal synthesis is associated with formation of well-crystallized MNPs, which in turn translates to increased saturation magnetization values [40]. In hydrothermal synthesis, the geometry of the nanoparticles is controlled by optimizing reaction parameters [37]. Indeed, nanoparticle size increases with prolonged reaction times and higher water content promotes particle aggregation [41]. Magnetite nanoparticles of narrow size distribution and high magnetic properties are synthesized by oxidation of $\mathrm{FeCl}_{2} \cdot 4 \mathrm{H}_{2} \mathrm{O}$ in basic aqueous media at $134^{\circ} \mathrm{C}$ [42]. Irregular and ellipsoid magnetite microtubes are obtained by neutral oxidation of $\mathrm{Fe}^{3+}$ and $\mathrm{Fe}^{2+}$ by $\mathrm{H}_{2} \mathrm{O}_{2}$, whereas magnetite nanotubes and nanoparticles are produced when $\mathrm{NH}_{4} \mathrm{HCO}_{3}$ and urea are used instead of $\mathrm{H}_{2} \mathrm{O}_{2}$ [43]. Furthermore, the hydrothermal technique can be utilized to synthesize magnetic composite particles, such as magnetite cores with silicon dioxide or titanium dioxide coating [44].

\section{Microemulsion}

Reverse 'micelle' microemulsion is another technique for MNP synthesis. Here, soluble metal salts $\left(\mathrm{Fe}^{2+} / \mathrm{Fe}^{3+}\right)$ are incorporated into aqueous microdroplets in oil that coalesce with hydroxide $\left(\mathrm{OH}^{-}\right)$-containing microdroplets to form magnetite-containing microdroplets. Particle size is a function of interdroplet

Table 1. Magnetic nanoparticle synthesis methods.

\begin{tabular}{|c|c|c|c|c|c|}
\hline Method $^{+}$ & Temperature & Simplicity & $\begin{array}{l}\text { Size } \\
\text { distribution }\end{array}$ & Yield & Coatings \\
\hline Co-precipitation & $\begin{array}{l}\text { Room } \\
\text { temperature }\end{array}$ & Simple, minutes & Narrow & High, scalable & $\begin{array}{l}\text { Dextran, chitosan, } \\
\text { other polymers }\end{array}$ \\
\hline $\begin{array}{l}\text { Hydrothermal } \\
\text { synthesis }\end{array}$ & High & $\begin{array}{l}\text { Simple, high } \\
\text { pressure, days }\end{array}$ & Very narrow & $\begin{array}{l}\text { Medium, } \\
\text { automated }\end{array}$ & $\begin{array}{l}\text { Silicon, titanium, } \\
\text { polyethylene glycol }\end{array}$ \\
\hline Microemulsion & $\begin{array}{l}\text { Room } \\
\text { temperature }\end{array}$ & Complicated, hours & Narrow & Low & Lipids, polymers \\
\hline $\begin{array}{l}\text { Thermal } \\
\text { decomposition }\end{array}$ & High & $\begin{array}{l}\text { Complicated, } \\
\text { inert conditions, } \\
\text { hours-days }\end{array}$ & Very narrow & High, scalable & $\begin{array}{l}\text { Chitosan, other } \\
\text { polymers, proteins, } \\
\text { surfactants }\end{array}$ \\
\hline
\end{tabular}


exchange and nuclei aggregation is affected by reaction temperature $[45,46]$. MNP synthesis by microemulsion can be accelerated by increased temperature [47]. Microemulsion is a method of choice for generating particles of narrow size distribution and is controlled by modulating the levels of aqueous droplets [48]. A proportional relationship between microdroplet size and molar water to surfactant ratio serves to control the particle size distribution [49]. MNPs produced by microemulsion are $<15 \mathrm{~nm}$ in size and show concordant chemical and physical properties [35]. The major drawbacks of microemulsion synthesis are low yield, difficulty in scale-up and difficulty in removing the surfactants bound to the particle surface [38]. However, microemulsion MNP synthesis offers the opportunity of simultaneous nanoparticle formation and polymerization of shell coats. MNPs of $80-180$-nm size can be synthesized by inverse microemulsion polymerization, while lower particle size is associated with increased surfactants and cross-linker concentration [50].

\section{Thermal decomposition}

Thermal decomposition provides good control over particle parameters [51]. Particle yield is high and scalable [52]. Thermal decomposition yields monodispersed magnetite $\left(\mathrm{Fe}_{3} \mathrm{O}_{4}\right)$, which can be further oxidized to form maghemite. Thermal decomposition can utilize iron pentacarbonyl $\left.\left(\mathrm{Fe}[\mathrm{CO}]_{5}\right]\right)$, as well as ferric acetylacetonate $\left(\mathrm{Fe}\left[\mathrm{C}_{5} \mathrm{H}_{7} \mathrm{O}_{2}\right]_{3}\right)$ as precursors. MNPs can be synthesized in the presence of organic surfactants such as oleic acid and/or oleylamine. Addition of oleic acid was reported to decrease particle size [53]. Thermal decomposition of $\mathrm{Fe}(\mathrm{CO})_{5}$ generates monodispersed oleic acid-coated magnetite nanoparticles of sizes smaller than $10 \mathrm{~nm}$ [51]. If thermal decomposition is carried out under air instead of inert conditions, maghemite particles are formed and the size can be specifically tuned between 3 and $17 \mathrm{~nm}$ [52]. Maghemite can also be synthesized by addition of the oxidizing agent trimethylamine- $N$-oxide [53].

Another method to tune the size of MNPs is separation of the nucleation and growth phases [26]. Prolonged thermal decomposition at low temperature contributes mainly to the nucleation phase, creating more nuclei and promoting formation of smaller sized particles [25]. Decomposition at higher temperature limits the nucleation phase, therefore promoting particle growth. Short nucleation time can be achieved by injecting cold precursors into a hot mixture of solvent and surfactants, therefore limiting the nucleation and promoting nanoparticle growth. Temperature and heating rates determine the size and shape of nanoparticles. Crystal/particle size increases with increased rate of heating from $0.4^{\circ} \mathrm{C} / \mathrm{min}$ to $3.5-4^{\circ} \mathrm{C} / \mathrm{min}$. At $3.5-4^{\circ} \mathrm{C} / \mathrm{min}$ growth phase begins to predominate over nucleation and size distribution increases from 5-7 nm to $10-13 \mathrm{~nm}$. Under these conditions, particle size was stable over $30 \mathrm{~min}$ [29,54].

\section{Particle coating \& surface stability}

Surface stabilization of the MNP core with polymer, silica, amino groups or other organic surfactants is required for functionality [55], as MNPs have an intrinsic tendency to aggregate due to their high surface energy and strong magnetic dipole-dipole interactions [56]. Moreover, electrostatic stabilization is not adequate to prevent aggregation in physiological solutions where salts tend to neutralize a surface charge. In vivo, MNP aggregates can clog capillaries [25]. MNP coating is an integral component of the design of the final nanoparticles system, as coating imparts colloidal stability and dispersal, prevents MNP surface oxidation, minimizes clearance by the RES and provides surface chemistry for bioconjugation of targeting ligands and drug molecules $[18,55,57]$. The literature contains a broad range of scientific approaches that serve to modify the MNP surface to facilitate translational clinical applications [21,58-60].

To surmount this obstacle, aqueous dispersion of MNPs is obtained by surface coating with hydrophilic polymers, such as polyethylene glycol (PEG), polyethyleneimine (PEI), dextran and chitosan, among others [61-63]. To date, several approaches are available to coat the MNP surface with appropriate polymers, including in situ and postsynthesis coating [21]. However, multiple anchoring groups on a polymer can bind more than one particle at a time leading to aggregates.

\section{Polyethylene glycol}

One of the most widely used approaches for the preparation of stable and biocompatible nanoparticles is to graft PEG onto the MNP surface, termed PEGylation. Because of their biocompatibility, PEGs are FDA-approved excipients in numerous pharmaceutical formulations [64-66]. Silane-coupling agents and other linking chemistries, such as 3 -aminopropyltrimethoxysilane, are commonly employed to immobilize PEG onto the MNP surface $[67,68]$. PEG layers can improve aqueous dispersity and its hydrophilic nature provides resistance to protein adsorption and particle opsonization, reducing RES clearance and thus increasing circulation time. PEG can be further grafted with targeting ligands to improve specific accumulation into tumors or other tissue sites of disease [69]. As an example, water-soluble magnetite nanoparticles were synthesized with monocarboxylterminated PEG allowing conjugation with anticarcinoembryonic antigen, a cancer targeting monoclonal 
antibody [70]. Recently, magnetic hybrid nanoparticles were made within a poly(lactic acid-co-glycolic acid) (PLGA) core, stabilized by PEG and lipid [71]. Notably, blood circulation half-life of meso-2,3-dimercaptosuccinic acid-coated MNP was increased twofold when conjugated to PEG [61].

\section{Dextran}

Dextrans are a class of biocompatible and biodegradable natural polysaccharides that are commonly used for coating MNPs. Dextrans are commonly employed for MNP coating because of their affinity for the iron oxide surface through chelation and hydrogen bonding. Carboxydextran and carboxymethyldextran are often used for MNP coating [18] because the length of the dextran chain and the large number of hydroxyl groups provide a high degree of hydrogen bonding [41]. Therefore, many FDA-approved MNPs are coated with dextran and its derivatives. To prevent desorption of the dextran, the coating can be crosslinked using epichlorohydrin and ammonia. However, although cross-linking with epichlorohydrin extends MNP circulation half-life, the nonbiodegradability of epichlorohydrin precludes use of these MNPs in clinical applications. As an alternative approach, covalent attachments to dextran on the MNP surface can be achieved by modifying the MNP surface with aminopropysilane groups [63]. Because of the large number of hydroxyl groups, the dextran coating can be readily functionalized with targeting moieties and fluorescent probes for specific delivery to sites of disease [63,72].

\section{Poly(lactic-co-glycolic acid)}

PLGA is a FDA-approved, biodegradable polymer, which has been used extensively for nanomedicine production, drug delivery and tissue engineering [73]. Furthermore, drug release rates from PLGA nanoparticles can be controlled, which is of importance for drug delivery [74]. Oleic acid-coated MNPs were incorporated into a PLGA matrix by an oil-in-water emulsion technique to serve as a delivery vehicle. Transmission electron microscopy demonstrated that MNPs smaller than $10 \mathrm{~nm}$ in size were dispersed uniformly throughout the PLGA matrix [51,75]. However, PLGA on the surface of MNPs decreased the saturation magnetization to approximately half of the original value [76].

\section{Polyethyleneimine}

PEI is a water soluble, cationic polymer that can be prepared in branched or linear form. Branched PEI of approximately $25 \mathrm{kDa}$ exhibit very high transfection efficiency and are considered as the 'gold standard' in the field of gene delivery [77]. PEI attachment onto the MNP surface can be performed by in situ coating, postsynthesis adsorption or grafting [18]. Capitalizing on these properties, PEI was recently used to modify MNPs for successful delivery of nucleic acids [78,79], where MNPs provided magnetic targeting, while PEI condensed the genetic material. These studies also demonstrated that PEI-coated MNPs improved delivery of encapsulated DNA to A549 and B16-F10 tumor cells in the presence of an external magnetic field [79]. In addition to gene transfection, PEI-modified MNPs have been used for delivery of a malaria DNA vaccine [80]. On the other hand, several problems such as its intrinsic toxicity and low colloidal stability restrict the use of PEI coating [81].

\section{Chitosan}

Chitosan is a biodegradable linear cationic polysaccharide formed by partial deacetylation of the natural polysaccharide chitin [46], which is extensively used in therapeutic applications. A two-step synthesis of biocompatible MNPs was developed to coat MNPs formed by co-precipitation with chitosan owing to the poor solubility of chitosan at the $\mathrm{pH}$ required for coprecipitation of MNPs [18]. First, MNPs were synthesized by co-precipitation of an aqueous salt solution of $\mathrm{Fe}^{2+}$ and $\mathrm{Fe}^{3+}$ with ammonia in the presence of a positively charged chitosan derivative. Second, an anionic derivative of chitosan was adsorbed onto the positively charged MNP, resulting in a negatively charged MNP [82]. The chitosan-coated MNP exhibited a very high saturation magnetization and formed a highly stable aqueous dispersion. For example, chitosan/PEGcoated MNPs were used for gene delivery, where the plasmid enhanced green fluorescence protein was encapsulated as a reporter gene [83]. Controlled DNA release from the nanoparticles was observed upon application of a magnetic field. Since chitosan possesses both amino and hydroxyl functional groups, functionalization of MNPs with imaging agents, targeting ligands and therapeutic agents for multimodule theranostic applications is possible [84].

\section{Silica}

Silica has numerous pharmaceutical applications. Silica coating of MNPs has been used to reduce oxidation of the magnetic core, which is a primary reason for loss of transverse relaxivity of the particles [78]. The silica shell can protect the metallic core from oxidation under aqueous conditions and silanol groups enable reaction with alcohols to provide stability in nonaqueous media and provide a platform for covalent linkage to numerous specific ligands [85]. Several methods have been reported to immobilize mesoporous silica onto the MNP surface facilitating drug 
delivery and imaging [86,87]. However, the particles that are generated are often hydrophobic [25]. As an alternative approach, a water-in-oil microemulsionbased synthesis has been used to generate particles of improved aqueous dispersity [88]. In this method, coating of silica onto the inner core of particles is controlled by micelle formation, and the aqueous domain is uniformly dispersed into a continuous oil phase and separated by a layer of surfactant. However, in situ microemulsion preparation generally leads to a poor crystalline magnetic core, limiting its applicability for production of clinically useful MNPs. Monodispersed silica-coated paramagnetic luminescent nanoparticles can be synthesized by microemulsion [89]. The most common synthetic procedures use a template, such as cetyltrimethylammonium bromide (CTAB), to grow long tubular structures upon which silica polymerizes to encapsulate MNP [90]. The CTAB template is subsequently removed by solvent extraction to yield MNPs with a highly ordered mesoporous silica coating. Mesoporous silica microspheres encapsulating a silica-coated MNP core were synthesized using the surfactant-template approach [90]. In these studies, magnetite particles coated with a silica layer were encapsulated within a mesostructured CTAB/silica template. Solvent extraction of CTAB yielded mesoporous silica microspheres containing MNPs. These MNP microspheres exhibited high magnetization and had uniform mesopores with a large pore volume. The inner silica core protects the metallic core from leaching, the outer mesoporous silica core provides a platform for functional derivatization and the large accessible pores can carry therapeutic or imaging cargos [90]. Of importance for imaging, silica coating enhances encapsulated fluorescent dye by preventing direct interaction with the metallic core and reducing luminescence quenching [91-93]. Scaled-up production of silica-coated MNPs is possible [94].

\section{Gold}

Fabrication of core-shell iron oxide/gold (Au) MNPs is achievable $[93,94]$. Au coating stabilizes the magnetite core by hampering oxidation and corrosion, and provides desired biocompatibility of the MNP. Au coating can also provide a surface for conjugation of a chemical or biological moiety through binding to thiol-terminate molecules. However, the thin Au coating does not prevent MNP aggregation, and thus an 'ionic capping ligand' is required during the coating process $[2,58]$. Au-coated MNPs using 4-benzylpyridine as a capping agent exhibit excellent stability in acidic conditions [95]. Au-coated MNPs can also be prepared by reduction of $\mathrm{Au}^{3+}$ onto the MNP surface [96]. Au-coated MNPs were prepared by reducing
$\mathrm{HAuCl}_{4}$ in a chloroform solution of magnetite in the presence of oleylamine, which served as both a surfactant and a mild reducing agent. The Au-coated MNPs were subsequently transferred to an aqueous solution of CTAB and sodium citrate for further coating by addition of $\mathrm{HAuCl}_{4}$ Similarly, a Au layer was coated onto amine-coated MNPs by sonicating MNPs in an aqueous solution of $\mathrm{HAuCl}_{4}$ in the presence of sodium citrate [97]. Au-coated MNPs can also be functionalized. Novel dumbbell-like targeted Au-coated MNPs were synthesized to effectively deliver platin to Her2positive breast cancer cells [98]. Core-shell composite Au-coated MNPs can be capped with CTAB to reduce aggregation by neutralizing the layer with a positive surface charge and stabilizing the core-shell nanoparticles. Polyacrylic acid can then be conjugated onto the CTAB cap using 1-ethyl-3-(3-dimethyllaminopropyl) carbodiimide as a cross-linker and the carboxylic group of polyacrylic acid can be used for conjugation of targeting moieties [99].

\section{Surface functionalization}

Targeted drug/gene delivery, which is achieved by targeting specific cell receptors, is key to reducing drug doses, improving drug efficacy and reducing unwanted toxicities [100]. In order to achieve this, MNP surface functionalization is critical [2]. As previously discussed, a protective coating can bestow physical and chemical colloidal stability and can also provide a platform for functionalization with specific targeting moieties $[100,101]$. Certain coatings such as $\mathrm{Au}$ and silica are excellent platforms for functionalization with targeting moieties due to their surface chemistry [55]. Numerous strategies have been utilized for tailoring the MNP surface to yield biocomposite nanoparticles with designated features required for biomedical applications [55]. The MNP surface can be functionalized with biological ligands by either covalent coupling via ester or amide linkages or by noncovalent grafting [21]. For covalent bonding, functional moieties are attached to the MNP surface using various linkers such as 1-ethyl-3-(3-dimethyllaminopropyl) carbodiimide/ $N$-hydroxysuccinimide, maleimide, pyridyl disulfide, or $N$-succinimidyl 3-(2-pyridyldithio) propionate [20]. Carbodiimide cross-linker chemistry is used for conjugation of a peptide or other moiety to the hydrophobic MNP surface via formation of an amide bond [58]. Biological ligands can be noncovalently grafted to MNPs through ionic bonding, physical adsorption or streptavidin conjugation [102] Ionic bonding can be manipulated chemically or by adjusting the ionic strength and/or $\mathrm{pH}$ of the solution. Although, noncovalent grafting of biomolecules offers a relatively easy approach, the bond can be unstable 
under physiological conditions, leading to detachment of the coating and precipitation of the MNP. Moreover, results of noncovalent grafting are often not reproducible and may produce erratic biological responses. To overcome these shortcomings, development of stable covalent linkages resistant to oxidation, hydrolysis and reducing conditions has gained more attention. However, a key concern during fabrication of functionalized biomagnetic nanoparticles is to preserve the activity and properties of the bioactive functional groups. Use of small molecules, in addition to increasing affinity through multivalent attachments, offers an advantage to preserving the functionality throughout synthesis [103]. By contrast, large molecules, such as proteins and antibodies, often lose functionality during the conjugation step. Conjugation of monoclonal antibodies to the MNP surface usually involves covalent binding of the most reactive amino groups present at the antigen-binding site (i.e., Fab). Furthermore, attachment may lead to random orientation of the antibodies, compromising their immune reactivity [104]. Several strategies have been employed to attach antibodies oriented in such a manner so as to maintain functionality, including use of immobilized protein [105] and immobilization through the sugar chain of the antibodies [106]. These approaches immobilize antibody onto the MNP surface through the Fc region, leaving the Fab region available for molecular recognition. A two-step approach for the attachment of antibody without loss of orientation was described [107]. The antibody is first adsorbed to the surface of the MNP via ionic adsorption, followed by an irreversible covalent attachment. Surface functionalization using antibodies has limitations that include instability with the polymeric coat, particle aggregation with small fluctuations in ionic strength and/or $\mathrm{pH}$, and unequal distribution over the MNP surface [107]. Moreover, conjugation of antibodies onto the MNP surface is usually a complex process due to the large size of the antibody, which impedes particle extravasation. Complicating matters, monoclonal antibodies are immunogenic in nature and can thus elicit an immune response that limits repeated application of the carrier system. Nevertheless, functionalization of MNPs with large proteins and antibodies is being explored for a variety of therapeutic applications. Liu et al. covalently attached antitumor antibody onto PEGylated MNPs using a 'click reaction' [108]. Others demonstrated good targeting efficiency of PEGylated MNPs functionalized with a monoclonal antibody to lymphatic vessel endothelial hyaluronan receptor-1 to mouse lymphatic endothelial cells [109]. In addition, accumulation of MNPs conjugated with luteinizing hormone-releasing hormone at the site of tumors that overexpress luteinizing hormone-releasing hormone receptors was demonstrated in breast and metastatic lung cancers, supporting the clinical applications of these particles [110].

The folate receptor binds folate and its conjugates with high affinity and is a common target for the management of various types of human cancers [111]. Folic acid-conjugated MNPs are a classic example of targeted delivery using MNPs. Folic acid-conjugated MNPs were used to target human nasopharyngeal epidermal carcinoma [72], breast cancer [112] and human hepatic carcinoma [113]. Multifunctional MNPs were produced where core-shell biodegradable magnetite nanospheres were conjugated to doxorubicin through a carboxyl linkage and functionalized with iminodiacetic acid-modified folate to provide tumor-targeting capability [114]. Other multifunctional MNPs have also been developed for targeted delivery of doxorubicin [115]. MNPs were coated with dual-responsive poly $(N$ isopropylacrylamide)-block-poly(acrylic acid) block copolymer, which enabled the sustained release of encapsulated doxorubicin at a desired temperature and low lysosomal pH. Cancer cell targeting was imparted to the design by tethering folic acid onto the surface of the MNP through an amide linkage.

Peptides can also be conjugated onto the surface of MNPs as functional moieties. The most commonly used peptide is arginine-glycine-aspartate, the recognition motif for integrin $\alpha_{v} \beta_{3}$ receptor, which is overexpressed in tumor blood vessels and some melanoma cancers [116]. Carboxymethyl dextran-coated ultrasmall MNPs conjugated with cyclic arginine-glycine-aspartate peptides exhibited higher uptake by Bcap37 cells compared with nontargeted MNPs [117]. In other studies, MNPs functionalized with a peptide targeted to the urokinase plasminogen activator receptor demonstrated fivefold greater uptake by urokinase plasminogen activator receptor-positive cells compared with untargeted MNPs [12]. Tumor-targeting capability of a magnetic nanovector consisting of a MNP core coated with copolymers of chitosan, PEG and PEI and conjugated with chlorotoxin was demonstrated by preferential accumulation in glioma cells in mice bearing C6 xenograft tumors [118]. In a unique application, MNPs functionalized with material-specific peptides were used to purify metals of interest from a colloidal mixture using an external magnetic field [119]. Thus, streptavidin-coated MNPs were modified with an Au-specific peptide to sequester Au nanoparticles from a colloidal mixture of Au and cadmium sulfide nanoparticles.

Conjugation of adenoviral vectors onto MNPs can provide concurrent MRI and gene delivery applications [55]. While adenoviral vectors offer the potential for vascular gene therapy, their rapid inactivation, 
suboptimal transduction efficiency and inherent immunogenicity, limit their applicability. The polymer coating of MNPs can protect the conjugated adenovirus from inactivation, and transfection efficiency can be enhanced by application of an external magnetic field to target the MNP to the desired site of action $[120,121]$. This was demonstrated in studies where successful transduction of endothelial and smooth muscle cells by adenovirus-conjugated MNPs occurred even after exposure to adenovirus-neutralizing antibodies and lithium iodide, an adenovirus disrupter agent [122]. Adenovirus-conjugated magnetic beads, under an external magnetic field, successfully transduced adenoviral vectors encoded with the $h V E G F$ gene for regeneration of ischemically damaged hearts in an acute myocardial infarction rat model [123]. Fabrication of biocomposite MNPs has also been described using other biomolecules such as bovine serum albumin [124], TAT peptide [125] and transferrin [126], each designed to impart a specific targeting efficacy to the particles.

\section{Design considerations}

The physicochemical characteristics of MNPs are of importance when designing a successful drug delivery system. Surface coating and functionalization of MNPs can be tailored to particular biomedical applications. Moreover, a biocomposite nanoconstruct requires that the biomolecule is biocompatible with biological systems and is not associated with any adverse effects. In addition, a biomolecule should impart adequate colloidal stability to allow the biocomposite MNP to reach its target $[25,37,55]$. A key fundamental consideration for synthesis of successful MNPs is discussed below.

\section{Geometry}

Size and shape are the key physical parameters that govern not only the colloidal stability of MNPs, but also influence the magnetic moment of the particles, and therefore the response to an external magnetic field [55]. For instance, saturation magnetization of MNPs is known to decrease with a decrease in particle size, a consequence of increased surface area that may have an impact on the magnetic moment [127]. However, particles smaller than $2 \mathrm{~nm}$ in size can diffuse through the cell membrane and damage intracellular organelles, and are thus not suitable for biomedical applications. The blood half-life of MNPs is determined in part by particle size. Particles smaller than $20 \mathrm{~nm}$ in size are excreted via the kidney, while particles greater than $200 \mathrm{~nm}$ in size accumulate in the spleen and the liver and are cleared from the circulation by tissue-resident macrophages [128]. Particles $10-200 \mathrm{~nm}$ in size exhibit longer circulation time by avoiding sequestration by the RES and are thus considered optimum for biomed- ical applications [129]. Smaller spherical nanoparticles concentrate at the center of blood vessels due to their higher diffusion rate, which restricts their interaction with endothelial cells and extends blood circulation time [18]. Moreover, nanoparticles of sizes smaller than $200 \mathrm{~nm}$ that avoid opsonization and RES uptake accumulate at a tumor site because of the enhanced permeation and retention effect $[130,131]$. Another key parameter that governs the biodistribution of MNPs is particle shape. Particle shape can influence the biodistribution and clearance of nanoparticles [132]. Huang et al. observed that rod-shaped and nonspherical nanoparticles showed prolonged systemic circulation compared with spherical nanoparticles. Moreover, they found that short rod-shaped mesoporous silica nanoparticles accumulated in the liver, whereas long rod-shaped nanoparticles accumulated in the spleen. Additionally, in contrast to long rod-shaped particles, short rod-shaped mesoporous silica nanoparticles were rapidly cleared from the circulation. These studies demonstrated that spherical nanoparticles are engulfed by phagocytic cells to a greater extent than nonspherical or rod-shaped nanoparticles in support of the longer circulation half-lives of nonspherical or rod-shaped nanoparticles. Moreover, shape directly influences cellular entry of nanoparticles. For instance, rod-shaped nanoparticles larger than $100 \mathrm{~nm}$ exhibit the highest cellular uptake, followed by spherical-, cylindrical- and cuboidal-shaped nanoparticles of similar sizes. However, for particle sizes smaller than $100 \mathrm{~nm}$, spherical nanoparticles exhibit preferentially higher cell uptake over rod-shaped nanoparticles [56].

\section{Surface charge}

Surface charge on MNPs is measured as the electrostatic potential at the surface of nanoparticles, termed 'zeta potential' or 'electrokinetic potential'. Zeta potential can indicate attachment of drug molecules onto the MNP surface and is a function of charge status of the drug moiety. It has been well established that the surface charge of MNPs determines the colloidal stability and influences biodistribution of nanoparticles. Following intravenous injection, MNPs encounter physiological conditions of higher ionic strength and a slightly basic $\mathrm{pH}(\mathrm{pH}$ 7.4). These unfavorable conditions, in combination with the inherent magnetic attraction of MNPs, induce particle aggregation. The hydrophobic and charged MNP surface undergoes nonspecific association with plasma proteins (i.e., opsonin) and extracellular matrix leading to RES clearance. These effects are counteracted by electrostatic repulsion induced by a charged particle surface, grafting a hydrophilic coating onto the particles and by coating the particles with a protective 
shell (steric barrier) [37,130]. The charge status of MNPs also impacts cellular internalization, thus influencing biodistribution. Positively charged nanoparticles show a higher rate of cellular uptake in comparison with negatively charged or neutral nanoparticles. As a consequence, positively charged nanoparticles have a relatively shorter blood half-life than negatively charged or neutral particles [133]. Positively charged MNPs are internalized by human breast cancer cells with higher efficiency compared with negatively charged MNPs. However, the uptake of MNPs can be cell type-specific as demonstrated by the lack of differential internalization of these particles by human umbilical vein endothelial cells [134].

Nonspecific adsorption of plasma proteins leads to clearance by the RES and is a function of the surface properties of nanoparticles including size, charge and hydrophilicity/hydrophobicity. Opsonization is greatly reduced for hydrophilic particles of small size [135]. To limit nanoparticle clearance by the RES, 'stealth' nanoparticles have been developed by coating the particles with PEG. PEGylation decreases particle interaction with the RES by reducing the potential for opsonization through steric hindrance [66]. Lee et al. reported that by using poly(3-[trimethoxysilyl propyl] methacrylate-r-polyethyleneglycol methacrylate) consisting of a surface anchoring moiety (silane group) and a "protein-resistant moiety" (PEG), to coat the magnetic core, protein- and cell- resistant MNP could be generated [59] that showed an extended circulation half-life.

\section{Surface thermodynamics \& colloidal stability}

Colloidal stability of MNPs in an aqueous solution is a prerequisite for biomedical applications. Interparticle interactions and wettability of particles in aqueous media govern the interfacial surface energy, which impacts particle aggregation and polydispersity. Moreover, the thermodynamic stability of MNPs in the absence of a magnetic field depends upon the balance between electrostatic forces, such as attractive forces (dipole-dipole and van der Waal interactions) and repulsive forces (steric and electrostatic). Electrostatic interaction can be adjusted by altering reaction parameters such as ionic strength and $\mathrm{pH}$. In an aqueous solution, hydrophobic MNPs tend to aggregate due to hydrophobic attraction. Therefore, grafting a hydrophilic moiety such as PEG onto the particle surface can provide adequate aqueous thermodynamic stability. Hydrophobic aggregation of MNPs is usually marked by a negative surface free energy, whereas making the MNP surface more hydrophilic through engraftment of a hydrophilic moiety is associated with positive free energy, which supports par- ticle dispersion and adequate aqueous stability. The moderate density of MNPs also supports resistance against gravitational settling in low blood flow $[37,136]$. Opsonization is influenced by the hydrophilic/hydrophobic nature of MNPs and occurs more rapidly with hydrophobic particles [137]. Adequate colloidal stability can thus be conferred by adsorption and/or grafting of surface stabilizers (e.g., PEG) onto the MNP surface. A sufficient stabilizer coating surrounding the MNP core can provide steric stabilization to overcome magnetic potentials and van der Waals attractive forces to impart long-term colloidal stability, even under harsh electrolyte, temperature and $\mathrm{pH}$ conditions [15,101,138].

The colloidal stability of MNPs can be monitored by periodic measurements of size as a function of electrolyte concentration and/or $\mathrm{pH}$ during a defined time period [139]. Aggregation of the particles can be determined by appearance of particles with larger hydrodynamic size. Moreover, long-term colloidal stability of MNPs under physiological conditions can be determined from temperature-dependent measurements of particle size using dynamic light scattering, which can provide a good estimation of the reversibility and binding affinity of the stabilizer coating on the MNP surface [140]. The colloidal stability of MNPs in solution can also be determined by analyzing the aggregation kinetics of MNPs using turbidity measurements [141].

\section{Drug encapsulation}

MNPs encapsulated with therapeutic cargo are efficient drug delivery vehicles, providing stabilization of the drug in physiological conditions, flexible drug loading and controlled drug release at the target site, while limiting nonspecific interactions with cells and other plasma proteins (Figure 2) [142-144]. The design of drug-loaded MNPs must consider all these factors. Of primary importance in constructing drug-loaded MNPs is that drug function should not be compromised during the loading process. Moreover, the drugloaded MNPs should encapsulate and protect a significant amount of therapeutic cargo, as determined by the method of drug loading and the nature of particle coating. Finally, drug-loaded MNPs should release their cargo in a controlled manner at the designated site for optimal efficacy. For instance, release of gene therapy cargo from MNPs can be tailored to respond to the cell cycle for optimal transfection [18].

Encapsulation of a drug onto MNPs can be achieved either by conjugation of the drug onto the surface via a physical or chemical linkage [145] or by encapsulating the drug into a composite polymer matrix [51]. A number of approaches have been utilized to encapsulate and/or conjugate drug or functional-targeting ligands onto the MNP surface, including conjugation 


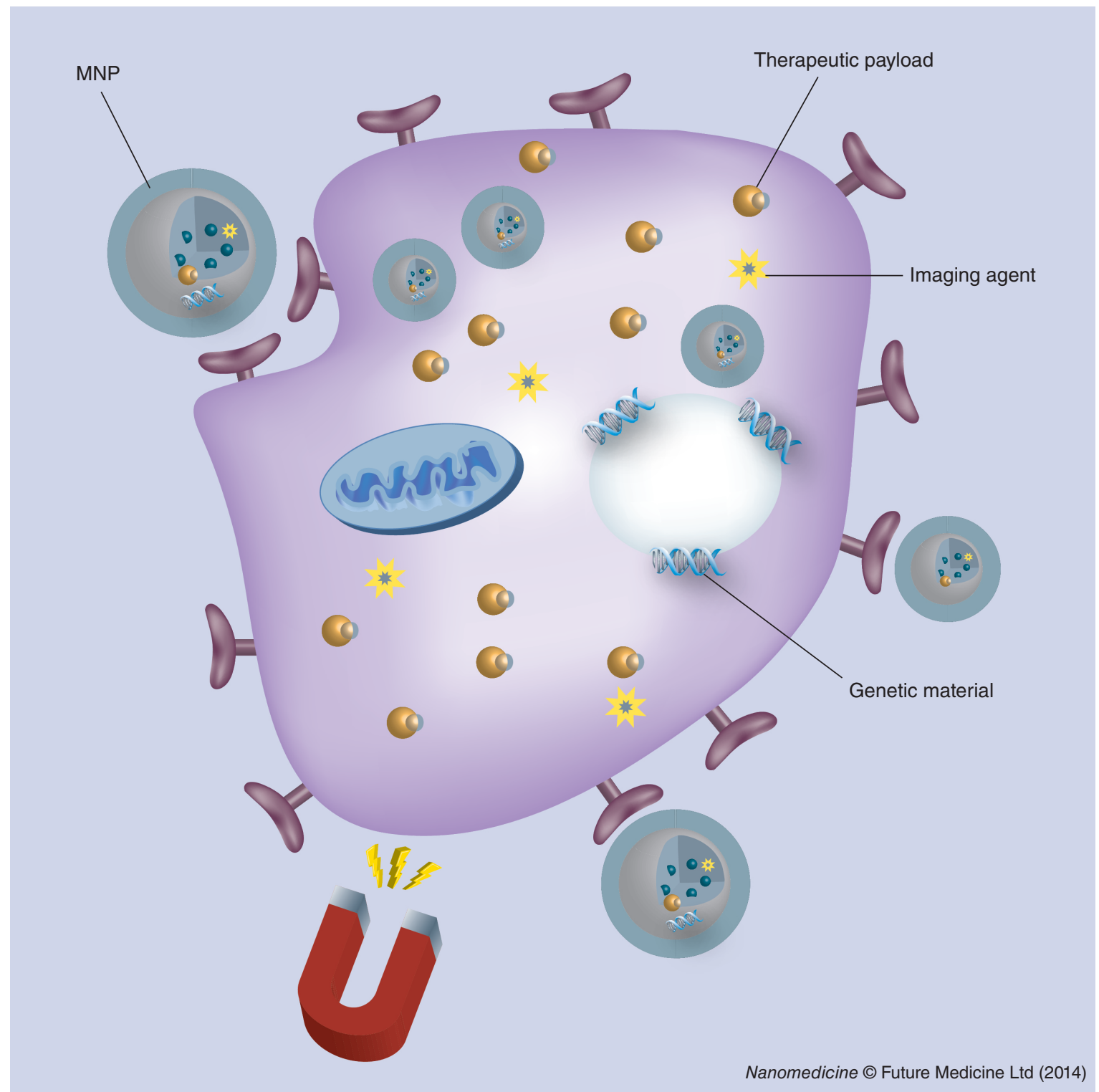

Figure 2. Cellular uptake of magnetic nanoparticles and intracellular release of therapeutic cargo under the influence of an applied magnetic field. Composite MNPs are shown with their ability to deliver a therapeutic payload to cells under the guidance of an external magnetic field. MNPs can be engineered with targeting molecules and/or cell penetrating peptides to promote efficient internalization.

MNP: Magnetic nanoparticle.

using cleavable covalent linkage and physical adsorption for intracellular release. Drug molecules are usually conjugated to the MNP surface through functional groups, such as thiol, hydroxyl and amino groups, on the polymer used to coat MNP, or conjugated to the MNP using cross-linkers, such as maleimide, and bifunctional cross-linkers, such as pyridyl disulfide $[51,145,146]$. Covalent linkage encapsulates the drug with high efficiency, while protecting the functionality and efficacy of the drug molecule. For encapsulation of peptides and proteins, which are susceptible to degradation under harsh oxidative conditions, milder conjugation reaction conditions need to be used. In some instances, however, a stable covalent linkage between the drug and the MNP can result in failure of drug release at the target site [146].

Drug molecules can be physically adsorbed onto the surface of MNP via electrostatic and affinity interactions [147]. This technique has been especially useful for encapsulation of nucleic acids. As an example, MNPs coated with a cationic lipid interlayer and coupled with negatively charged plasmid DNA via electrostatic interaction successfully delivered nucleic acids to a target site using magnetic transfection [147]. Similarly, MNPs coated within PEI, a cationic polymer, successfully condensed negatively charged 
genetic material and improved the transfection efficiency of the carrier system under the influence of an external magnetic field [79]. Hydrophobic drugs can also be encapsulated via hydrophobic interactions. Thus, MNPs coated with a hydrophobic polymer matrix can encapsulate drugs by hydrophobic interaction and then release the loaded drugs upon degradation of the polymer shell [146]. Affinity interactions, such as streptavidin-biotin bioconjugation, are also employed to couple the drug molecules or targeting ligands with MNPs. Contrary to hydrophobic and electrostatic interaction, affinity interaction is a stable noncovalent linkage that provides improved stability in a variety of electrolyte and $\mathrm{pH}$ conditions [148]. The downside of physical conjugation of drugs onto the MNP surface is poor drug entrapment efficiency [146]. To overcome low entrapment efficiency, drug molecules can be encapsulated into the polymer matrix along with MNPs [51,149]. Moreover, magnetoliposomes, which represent a novel class of magnetic composite nanoconstructs, offer a platform to ensure higher entrapment of both MNPs and drug molecules, while providing an optimum magnetic response and maintaining therapeutic efficacy of the encapsulated cargo $[150,151]$.

\section{Magnetism}

Several excellent reviews have been published to elaborate on the magnetic characteristics of MNPs $[2,25,37,55,152]$. In the present article, we will limit our discussion to a brief overview of the superparamagnetic behavior of MNPs. Superparamagnetism is required for MNPs to be useful for biomedical applications [153]. Superparamagnetism is a type of magnetism, which occurs in $<50 \mathrm{~nm}$, single-domain nanoparticles, where the total magnetic moment of the nanoparticle is a summary of magnetic moments of all the atoms comprising the nanoparticle [153].

The magnetic property of any material is described by the significance of correlation between magnetic induction (B) and magnetic field (H) [37,153,154]. In most MNPs, the dependence of magnetic induction on the magnetic field can be best described by a linear relationship between $\mathrm{B}$ and $\mathrm{H} ; \mathrm{B}=\mu \mathrm{H}$, where $\mu$ is the magnetic permeability of the MNP. A MNP exhibits paramagnetism in the case of $\mu>1$, and diamagnetism in the case of $\mu<1$. Alternatively, magnetic susceptibility $(\chi=\mu-1)$ can be used to describe magnetism in MNPs; for paramagnetic MNPs $\chi>0$, and for diamagnetic particles $\chi<0$ [37,58]. Ferromagnetic particles, which usually have an unpaired electron spin, exhibit permanent magnetization in the absence of an external magnetic field. However, once ground down to a dimension $<30 \mathrm{~nm}$, particles become single- domain particles and become superparamagnetic. For superparamagnetic nanoparticles, no permanent magnetism remains upon suppression of an external magnetic field, which reduces aggregation, extends blood circulation half-life and avoids formation of embolisms in blood vessels. However, although there is an increase in superparamagnetic behaviors with reduction in size, saturation magnetism of MNPs decreases with particle sizes $<10 \mathrm{~nm}$, and this adversely affects the susceptibility of the particle to magnetic targeting, as well as the relaxivity and thus efficiency of MNPs for biomedical applications. Decreased particle size has a significant impact on surface effects such as spin-canting, noncollinear spin and spin-glass-like behavior, which results in an alteration of magnetic properties. Moreover, MNPs obtained from different synthetic procedures display significant variations in their magnetic properties, such as saturation magnetization. These differences have been attributed to the crystal structure, impurities and surface coating. Superparamagnetic MNPs, in addition to their desired magnetic properties, also offer an advantage of heating behavior that comes from the Neel relaxation mechanism, which is widely employed in the field of hyperthermia $[37,154]$.

\section{Conclusion \& future perspective}

Recent medical research suggests that MNP-based biocomposites can be a promising platform for numerous in vitro and in vivo biological applications. The versatility of MNP-based nanotechnology is attributed to their capability to respond to an external magnetic force, and in most cases, their inherent ability to be functionalized with bioactive moieties. To date, MNPs are widely accepted for human use as MRI contrast agents, with formulations such as Feridex IV ${ }^{\circledR}$, Endorem $^{\circledR}$, Combidex $^{\circledR}$, Lumiren ${ }^{\circledR}$ (all from Advanced Magnetic [155-157].

\section{Acknowledgements}

The authors thank Tatiana Bronich for critical reading of the manuscript, sharing of noteworthy ideas and lively and continuous discussions.

Financial \& competing interests disclosure This work was supported by the US NIH grants 1P01DA028555, 2R01NS034239, 2R01NS36126, P01NS43985, P30RR031151 and P01MH64570. The authors have no other relevant affiliations or financial involvement with any organization or entity with a financial interest in or financial conflict with the subject matter or materials discussed in the manuscript apart from those disclosed.

No writing assistance was utilized in the production of this manuscript. 
Executive summary

Magnetic nanoparticles

- Nanomedicine facilitates development of nanoparticles using a range of therapeutic materials for improved diagnosis, targeting and treatment of disease.

- The versatility of magnetic nanoparticles (MNPs) results from their ability to respond to an external magnetic field.

Formulation developments \& product synthesis

- Substantial progress has been made in the synthesis of high-quality MNPs, covering a range of tunable composition, size and magnetic properties.

- Co-precipitation and thermal decomposition have been the methods of choice to generate MNP particles of reproducible composition and high yield.

Surface functionalization

- MNP surface functionalization is considered critical for biomedical applications.

- Surface functionalization bestows not only physical and chemical colloidal stability to MNPs, but can also provide a means of targeting specific tissue and disease sites.

- The MNP surface can be coupled to biological ligands by either chemical bonding or noncovalent grafting of biological ligands.

\section{Design considerations}

- Superparamagnetism is a key requisite for MNPs to be employed for biomedical applications.

- The geometry of MNPs governs colloidal stability, biodistribution and magnetic moment of the particles.

- The surface characteristics of MNPs are important determinants of colloidal stability and biodistribution. Hydrophobic and charged MNP surfaces undergo nonspecific association with plasma proteins (e.g., opsonin), which leads to clearance by the reticuloendothelial system.

\section{References}

Papers of special note have been highlighted as:

- of interest

1 Kim BY, Rutka JT, Chan WC. Nanomedicine. N. Engl. J. Med. 363(25), 2434-2443 (2010).

2 Lu AH, Salabas EL, Schuth F. Magnetic nanoparticles: synthesis, protection, functionalization, and application. Angew. Chem. Int. Ed. Engl. 46(8), 1222-1244 (2007).

- Presents extensive discussion on the synthesis for magnetic nanoparticles (MNPs).

3 Zhang L, Wang Y, Tang Y et al. High MRI performance fluorescent mesoporous silica-coated magnetic nanoparticles for tracking neural progenitor cells in an ischemic mouse model. Nanoscale 5(10), 4506-4516 (2013).

4 Sahin F, Turan E, Tumturk H, Demirel G. Core-shell magnetic nanoparticles: a comparative study based on silica and polydopamine coating for magnetic bio-separation platforms. Analyst 137(23), 5654-5658 (2012).

5 Sadhukha T, Wiedmann TS, Panyam J. Inhalable magnetic nanoparticles for targeted hyperthermia in lung cancer therapy. Biomaterials 34(21), 5163-5171 (2013).

6 Zheng J, Wang J, Tang T, Li G, Cheng H, Zou S. Experimental study on magnetic drug targeting in treating cholangiocarcinoma based on internal magnetic fields. Chin. Ger. J. Clin. Oncol. 5(5), 336-338 (2006).

7 Artemov D, Mori N, Okollie B, Bhujwalla ZM. MR molecular imaging of the Her-2/neu receptor in breast cancer cells using targeted iron oxide nanoparticles. Magn. Reson. Med. 49(3), 403-408 (2003).

8 Sensenig R, Sapir Y, MacDonald C, Cohen S, Polyak B. Magnetic nanoparticle-based approaches to locally target therapy and enhance tissue regeneration in vivo. Nanomedicine (Lond.) 7(9), 1425-1442 (2012).
9 Jafari T, Simchi A, Khakpash N. Synthesis and cytotoxicity assessment of superparamagnetic iron-gold core-shell nanoparticles coated with polyglycerol. J. Coll. Int. Sci. 345(1), 64-71 (2010).

10 Beduneau A, Ma Z, Grotepas CB et al. Facilitated monocyte-macrophage uptake and tissue distribution of superparmagnetic iron-oxide nanoparticles. PLoS ONE 4(2), e4343 (2009).

11 Chatterjee J, Haik Y, Chen C-J. Size dependent magnetic properties of iron oxide nanoparticles. J. Magn. Magn. Mat. 257(1), 113-118 (2003).

12 Hansen L, Larsen EK, Nielsen EH et al. Targeting of peptide conjugated magnetic nanoparticles to urokinase plasminogen activator receptor (uPAR) expressing cells. Nanoscale 5(17), 8192-8201 (2013).

13 Kievit FM, Zhang M. Surface engineering of iron oxide nanoparticles for targeted cancer therapy. Acc. Chem. Res. 44(10), 853-862 (2011).

14 Mahajan SD, Aalinkeel R, Law WC et al. Anti-HIV-1 nanotherapeutics: promises and challenges for the future. Int. J. Nanomed. 7, 5301-5314 (2012).

15 Amstad E, Textor M, Reimhult E. Stabilization and functionalization of iron oxide nanoparticles for biomedical applications. Nanoscale 3(7), 2819-2843 (2011).

16 Weissleder R, Hahn PF, Stark DD et al. MR imaging of splenic metastases: ferrite-enhanced detection in rats. $A J R$ Am. J. Roentgenol. 149(4), 723-726 (1987).

17 Krishnan KM. Biomedical nanomagnetics: a spin through possibilities in imaging, diagnostics, and therapy. IEEE Transact. Magnet. 46(7), 2523-2558 (2010).

18 Veiseh O, Gunn JW, Zhang M. Design and fabrication of magnetic nanoparticles for targeted drug delivery and imaging. Adv. Drug Deliv. Rev. 62(3), 284-304 (2010). 
- Considers current status and future uses of MNPs with an eye to clinical translation.

19 Hutten A, Sudfeld D, Ennen I et al. New magnetic nanoparticles for biotechnology. J. Biotechnol. 112(1-2), 47-63 (2004).

Lattuada M, Hatton TA. Functionalization of monodisperse magnetic nanoparticles. Langmuir 23(4), 2158-2168 (2007).

21 Gupta AK, Naregalkar RR, Vaidya VD, Gupta M. Recent advances on surface engineering of magnetic iron oxide nanoparticles and their biomedical applications. Nanomedicine (Lond.) 2(1), 23-39 (2007).

22 Domingo C, Rodriguez-Clemente Rl, Blesa M. Morphological properties of $\alpha-\mathrm{FeOOH}, \gamma-\mathrm{FeOOH}$ and $\mathrm{Fe}_{3} \mathrm{O}_{4}$ obtained by oxidation of aqueous $\mathrm{Fe}$ (II) solutions. J. Coll. Int. Sci. 165(1), 244-252 (1994).

23 Cornell RM, Schwertmann U. The Iron Oxides: Structure, Properties, Reactions, Occurrences and Uses (2nd Edition). Wiley-VCH, Germany (2003).

24 LaMer VK, Dinegar RH. Theory, production and mechanism of formation of monodispersed hydrosols. J. Am. Chem. Soc. 72(11), 4847-4854 (1950).

- Describes the basic fundamentals of monodispersed nanoparticles.

25 Schladt TD, Schneider K, Schild H, Tremel W. Synthesis and bio-functionalization of magnetic nanoparticles for medical diagnosis and treatment. Dalton Trans. 40(24), 6315-6343 (2011).

26 Alivisatos AP. Perspectives on the physical chemistry of semiconductor nanocrystals. J. Phys. Chem. 100(31), 13226-13239 (1996).

27 Hyeon T. Chemical synthesis of magnetic nanoparticles. Chem. Commun. (Camb.) 8, 927-934 (2003).

- Presents a detailed study of the synthetic procedures used for the development of MNPs.

28 Cheon J, Kang NJ, Lee SM, Lee JH, Yoon JH, Oh SJ. Shape evolution of single-crystalline iron oxide nanocrystals. J. Am. Chem. Soc. 126(7), 1950-1951 (2004).

29 Park J, An K, Hwang Y et al. Ultra-large-scale syntheses of monodisperse nanocrystals. Nat. Mat. 3(12), 891-895 (2004).

30 Baumgartner J, Bertinetti L, Widdrat M, Hirt AM, Faivre D. Formation of magnetite nanoparticles at low temperature: from superparamagnetic to stable single domain particles. PLoS ONE 8(3), e57070 (2013).

31 Fried T, Shemer G, Markovich G. Ordered two-dimensional arrays of ferrite nanoparticles. Adv. Mater. 13(15), 1158-1161 (2001).

32 Neveu S, Bee A, Robineau M, Talbot D. Size-selective chemical synthesis of tartrate stabilized cobalt ferrite ionic magnetic fluid. J. Coll. Int. Sci. 255(2), 293-298 (2002).

33 Tartaj P, Morales MP, Veintemillas-Verdaguer S, GonzalezCarreño T, Serna CJ. Chapter 5 synthesis, properties and biomedical applications of magnetic nanoparticles. Handbook Mag. Mater. 16, 403-482 (2006).

34 Jolivet J, Belleville P, Tronc E, Livage J. Influence of Fe(II) on the formation of the spinel iron oxide in alkaline medium. Clay Clay Miner. 40 (5), 531-531 (1992).
35 Gupta AK, Wells S. Surface-modified superparamagnetic nanoparticles for drug delivery: preparation, characterization, and cytotoxicity studies. IEEE Transact. Nanobiosci. 3(1), 66-73 (2004).

36 Lin H, Watanabe Y, Kimura M, Hanabusa K, Shirai H. Preparation of magnetic poly (vinyl alcohol)(PVA) materials by in situ synthesis of magnetite in a PVA matrix. J. Appl. Polym. Sci. 87(8), 1239-1247 (2003).

37 Reddy LH, Arias JL, Nicolas J, Couvreur P. Magnetic nanoparticles: design and characterization, toxicity and biocompatibility, pharmaceutical and biomedical applications. Chem. Rev. 112(11), 5818-5878 (2012).

38 Tran PH, Tran TT, Vo TV, Lee BJ. Promising iron oxidebased magnetic nanoparticles in biomedical engineering. Arch. Pharm. Res. 35(12), 2045-2061 (2012).

39 Cansell F, Chevalier B, Demourgues A et al. Supercritical fluid processing: a new route for materials synthesis. J. Mater. Chem. 9(1), 67-75 (1999).

40 Wang J, Sun J, Sun Q, Chen Q. One-step hydrothermal process to prepare highly crystalline $\mathrm{Fe}_{3} \mathrm{O}_{4}$ nanoparticles with improved magnetic properties. Mater. Res. Bull. 38(7), 1113-1118 (2003).

41 Laurent S, Forge D, Port M et al. Magnetic iron oxide nanoparticles: synthesis, stabilization, vectorization, physicochemical characterizations, and biological applications. Chem. Rev. 108(6), 2064-2110 (2008).

$42 \mathrm{Ge} S$, Shi X, Sun K et al. A facile hydrothermal synthesis of iron oxide nanoparticles with tunable magnetic properties. J. Phys. Chem. C Nanomat. Int. 113(31), 13593-13599 (2009).

43 Li X, Zhang F, Ma C, Saul E, He N. Green synthesis of uniform magnetite $\left(\mathrm{Fe}_{3} \mathrm{O}_{4}\right)$ nanoparticles and micron cubes. J. Nanosci. Nanotechnol. 12(3), 2939-2942 (2012).

44 Linley S, Leshuk T, Gu FX. Synthesis of magnetic rattle-type nanostructures for use in water treatment. ACS Appl. Mat. Int. 5(7), 2540-2548 (2013).

45 Gibaud S, Attivi D. Microemulsions for oral administration and their therapeutic applications. Expert Opin. Drug Deliv. 9(8), 937-951 (2012).

46 Lin MM, Kim do K, El Haj AJ, Dobson J. Development of superparamagnetic iron oxide nanoparticles (SPIONS) for translation to clinical applications. IEEE Transact. Nanobiosci. 7(4), 298-305 (2008).

47 Chen D-H, Wu S-H. Synthesis of nickel nanoparticles in water-in-oil microemulsions. Chem. Mater. 12(5), 1354-1360 (2000).

48 Munshi N, De TK, Maitra A. Size modulation of polymeric nanoparticles under controlled dynamics of microemulsion droplets. J. Coll. Int. Sci. 190(2), 387-391 (1997).

49 Paul BK, Moulik SP. Uses and applications of microemulsions. Curr. Sci. India 80(8), 990-1001 (2001).

50 Deng Y, Wang L, Yang W, Fu S, Elaıssari A. Preparation of magnetic polymeric particles via inverse microemulsion polymerization process. J. Mag. Mag. Mater. 257(1), 69-78 (2003).

51 Kirthivasan B, Singh D, Bommana MM, Raut SL, Squillante E, Sadoqi M. Active brain targeting of a fluorescent P-gp 
substrate using polymeric magnetic nanocarrier system. Nanotechnology 23(25), 255102 (2012).

52 De Berti I, Cagnoli M, Pecchi G et al. Alternative lowcost approach to the synthesis of magnetic iron oxide nanoparticles by thermal decomposition of organic precursors. Nanotechnology 24(17), 175601 (2013).

53 Hyeon T, Lee SS, Park J, Chung Y, Na HB. Synthesis of highly crystalline and monodisperse maghemite nanocrystallites without a size-selection process. J. Am. Chem. Soc. 123(51), 12798-12801 (2001).

54 Belaid S, Laurent S, Vermeech M, Vander Elst L, PerezMorga D, Muller RN. A new approach to follow the formation of iron oxide nanoparticles synthesized by thermal decomposition. Nanotechnology 24(5), 055705 (2013).

55 Colombo M, Carregal-Romero S, Casula MF et al. Biological applications of magnetic nanoparticles. Chem. Soc. Rev. 41(11), 4306-4334 (2012).

- Presents an extensive overview of fundamental aspects of MNPs important for optimizing their biomedical applications.

56 Albanese A, Tang PS, Chan WC. The effect of nanoparticle size, shape, and surface chemistry on biological systems. Ann. Rev. Biomed. Eng. 14, 1-16 (2012).

57 Sun C, Lee JS, Zhang M. Magnetic nanoparticles in MR imaging and drug delivery. Adv. Drug Deliv. Rev. 60(11), 1252-1265 (2008).

58 Gupta AK, Gupta M. Synthesis and surface engineering of iron oxide nanoparticles for biomedical applications. Biomaterials 26(18), 3995-4021 (2005).

59 Lee H, Lee E, Kim do K, Jang NK, Jeong YY, Jon S. Antibiofouling polymer-coated superparamagnetic iron oxide nanoparticles as potential magnetic resonance contrast agents for in vivo cancer imaging. J. Am. Chem. Soc. 128(22), 7383-7389 (2006).

60 Lu Y, Yin Y, Mayers BT, Xia Y. Modifying the surface properties of superparamagnetic iron oxide nanoparticles through a sol-gel approach. Nano Lett. 2(3), 183-186 (2002).

61 Ruiz A, Hernandez Y, Cabal C et al. Biodistribution and pharmacokinetics of uniform magnetite nanoparticles chemically modified with polyethylene glycol. Nanoscale 5(23), 11400-11408 (2013).

62 Li J, Zheng L, Cai $\mathrm{H}$ et al. Polyethyleneimine-mediated synthesis of folic acid-targeted iron oxide nanoparticles for in vivo tumor MR imaging. Biomaterials 34(33), 8382-8392 (2013).

63 Mornet S, Portier J, Duguet E. A method for synthesis and functionalization of ultrasmall superparamagnetic covalent carriers based on maghemite and dextran. J. Magn. Magn. Mater. 293(1), 127-134 (2005).

64 Knop K, Hoogenboom R, Fischer D, Schubert US. Poly(ethylene glycol) in drug delivery: pros and cons as well as potential alternatives. Angew. Chem. Int. Ed. Engl. 49(36), 6288-6308 (2010).

65 Fuertges F, Abuchowski A. The clinical efficacy of poly(ethylene glycol)-modified proteins. J. Control. Release 11(1), 139-148 (1990).
66 Harris JM, Chess RB. Effect of pegylation on pharmaceuticals. Nat. Rev. Drug Discov. 2(3), 214-221 (2003).

67 Kim D, Toprak M, Mikhailova M. Surface modification of superparamagnetic nanoparticles for in vivo biomedical applications. MRS Proceedings 704, W11.2.1-W11.2.6 (2001).

68 Lutz JF, Stiller S, Hoth A, Kaufner L, Pison U, Cartier R. One-pot synthesis of pegylated ultrasmall iron-oxide nanoparticles and their in vivo evaluation as magnetic resonance imaging contrast agents. Biomacromolecules 7(11), 3132-3138 (2006).

69 Xie J, Xu C, Kohler N, Hou Y, Sun S. Controlled PEGylation of monodisperse $\mathrm{Fe} 3 \mathrm{O} 4$ nanoparticles for reduced nonspecific uptake by macrophage cells. Adv. Mater. 19(20), 3163-3166 (2007).

70 Li Z, Wei L, Gao MY, Lei HY. One-pot reaction to synthesize biocompatible magnetite nanoparticles. $A d v$. Mater. 17(8), 1001-1005 (2005).

71 Aryal S, Key J, Stigliano C, Ananta JS, Zhong M, Decuzzi P. Engineered magnetic hybrid nanoparticles with enhanced relaxivity for tumor imaging. Biomaterials 34(31), 7725-7732 (2013)

72 Choi H, Choi SR, Zhou R, Kung HF, Chen IW. Iron oxide nanoparticles as magnetic resonance contrast agent for tumor imaging via folate receptor-targeted delivery. Acad. Radiol. 11(9), 996-1004 (2004).

73 Sah H, Thoma LA, Desu HR, Sah E, Wood GC. Concepts and practices used to develop functional PLGA-based nanoparticulate systems. Int. J. Nanomed. 8, 747-765 (2013).

74 Danhier F, Ansorena E, Silva JM, Coco R, Le Breton A, Preat V. PLGA-based nanoparticles: an overview of biomedical applications. J. Control. Release 161(2), 505-522 (2012).

75 Raut SL, Kirthivasan B, Bommana MM, Squillante E, Sadoqi M. The formulation, characterization and in vivo evaluation of a magnetic carrier for brain delivery of NIR dye. Nanotechnology 21(39), 395102 (2010).

76 Gomez-Lopera S, Plaza R, Delgado A. Synthesis and characterization of spherical magnetite/biodegradable polymer composite particles. J. Coll. Int. Sci. 240(1), 40-47 (2001).

77 Patnaik S, Gupta KC. Novel polyethylenimine-derived nanoparticles for in vivo gene delivery. Expert Opin. Drug Deliv. 10(2), 215-228 (2013).

78 Hurley KR, Lin YS, Zhang J, Egger SM, Haynes CL. Effects of mesoporous silica coating and post-synthetic treatment on the transverse relaxivity of iron oxide nanoparticles. Chem. Mat. 25(9), 1968-1978 (2013).

79 Zhou Y, Tang Z, Shi C, Shi S, Qian Z, Zhou S. Polyethylenimine functionalized magnetic nanoparticles as a potential non-viral vector for gene delivery. J. Mat. Sci. Mat. Med. 23(11), 2697-2708 (2012).

80 Nawwab Al-Deen F, Ma C, Xiang SD, Selomulya C, Plebanski M, Coppel RL. On the efficacy of malaria DNA vaccination with magnetic gene vectors. J. Control. Release 168(1), 10-17 (2013). 
81 Petri-Fink A, Steitz B, Finka A, Salaklang J, Hofmann H. Effect of cell media on polymer coated superparamagnetic iron oxide nanoparticles (SPIONs): colloidal stability, cytotoxicity, and cellular uptake studies. Eur. J. Pharm. Biopharm. 68(1), 129-137 (2008).

82 Szpak A, Kania G, Skorka T, Tokarz W, Zapotoczny S, Nowakowska M. Stable aqueous dispersion of superparamagnetic iron oxide nanoparticles protected by charged chitosan derivatives. J. Nanopart. Res. 15(1), 1372 (2013).

83 Kuang Y, Yuan T, Zhang Z, Li M, Yang Y. Application of ferriferous oxide modified by chitosan in gene delivery. J. Drug Deliv. 2012, 920764 (2012).

84 Cumpstey I. Chemical modification of polysaccharides. ISRN Organic Chem. 2013, 417672 (2013).

85 Liu Y, Lou C, Yang H, Shi M, Miyoshi H. Silica nanoparticles as promising drug/gene delivery carriers and fluorescent nanoprobes: recent advances. Curr. Cancer Drug Target. 11(2), 156-163 (2011).

86 Lee JE, Lee N, Kim T, Kim J, Hyeon T. Multifunctional mesoporous silica nanocomposite nanoparticles for theranostic applications. Acc. Chem. Res. 44(10), 893-902 (2011).

87 Gordon R, Hogan CE, Neal ML, Anantharam V, Kanthasamy AG, Kanthasamy A. A simple magnetic separation method for high-yield isolation of pure primary microglia. J. Neurosci. Method. 194(2), 287-296 (2011).

88 Santra S, Tapec R, Theodoropoulou N, Dobson J, Hebard A, Tan W. Synthesis and characterization of silica-coated iron oxide nanoparticles in microemulsion: the effect of nonionic surfactants. Langmuir 17, 2900-2906 (2001).

89 Lipani E, Laurent S, Surin M, Vander Elst L, Leclere P, Muller RN. High-relaxivity and luminescent silica nanoparticles as multimodal agents for molecular imaging. Langmuir 29(10), 3419-3427 (2013).

90 Deng Y, Qi D, Deng C, Zhang X, Zhao D.

Superparamagnetic high-magnetization microspheres with an $\mathrm{Fe}_{3} \mathrm{O}_{4} @ \mathrm{SiO}_{2}$ core and perpendicularly aligned mesoporous $\mathrm{SiO} 2$ shell for removal of microcystins. J. Am. Chem. Soc. 130(1), 28-29 (2008).

91 Santra S, Yang H, Dutta D et al. TAT conjugated, FITC doped silica nanoparticles for bioimaging applications. Chem. Commun. (Camb.) 24, 2810-2811 (2004).

92 Fuller JE, Zugates GT, Ferreira LS et al. Intracellular delivery of core-shell fluorescent silica nanoparticles. Biomaterials 29(10), 1526-1532 (2008).

93 Burns AA, Vider J, Ow H et al. Fluorescent silica nanoparticles with efficient urinary excretion for nanomedicine. Nano Lett. 9(1), 442-448 (2008).

$94 \mathrm{Qu} \mathrm{H}$, Tong S, Song K et al. Controllable in situ synthesis of magnetite coated silica-core water-dispersible hybrid nanomaterials. Langmuir 29(33), 10573-10578 (2013).

95 Ban Z, Barnakov YA, Li F, Golub VO, O'Connor CJ. The synthesis of core-shell iron@gold nanoparticles and their characterization. J. Mater. Chem. 15(43), 4660-4662 (2005).

$96 \mathrm{Xu} Z$, Hou Y, Sun S. Magnetic core/shell $\mathrm{Fe}_{3} \mathrm{O}_{4} / \mathrm{Au}$ and $\mathrm{Fe}_{3} \mathrm{O}_{4} / \mathrm{Au} / \mathrm{Ag}$ nanoparticles with tunable plasmonic properties. J. Am. Chem. Soc. 129 (28), 8698-8699 (2007).
97 Smolensky ED, Neary MC, Zhou Y, Berquo TS, Pierre VC. $\mathrm{Fe}_{3} \mathrm{O}_{4} @$ organic@Au: core-shell nanocomposites with high saturation magnetisation as magnetoplasmonic MRI contrast agents. Chem. Commun. (Camb.) 47(7), 2149-2151 (2011).

$98 \mathrm{Xu}$ C, Wang B, Sun S. Dumbbell-like Au- $\mathrm{Fe}_{3} \mathrm{O}_{4}$ nanoparticles for target-specific platin delivery. J. Am. Chem. Soc. 131(12), 4216-4217 (2009).

99 Yang D, Ma J, Zhang Q et al. Polyelectrolyte-coated gold magnetic nanoparticles for immunoassay development: toward point of care diagnostics for syphilis screening. Analyt. Chem. 85(14), 6688-6695 (2013).

100 Emerich DF, Thanos CG. Targeted nanoparticle-based drug delivery and diagnosis. J. Drug Target. 15(3), 163-183 (2007).

101 Di Marco M, Guilbert I, Port M, Robic C, Couvreur P, Dubernet C. Colloidal stability of ultrasmall superparamagnetic iron oxide (USPIO) particles with different coatings. Int. J. Pharmaceut. 331(2), 197-203 (2007).

102 Jung HI, Kettunen MI, Davletov B, Brindle KM. Detection of apoptosis using the C2A domain of synaptotagmin I. Bioconjug. Chem. 15(5), 983-987 (2004).

103 Weissleder R, Kelly K, Sun EY, Shtatland T, Josephson L. Cell-specific targeting of nanoparticles by multivalent attachment of small molecules. Nat. Biotechnol. 23(11), 1418-1423 (2005).

104 Chou SW, Shau YH, Wu PC, Yang YS, Shieh DB, Chen CC. In vitro and in vivo studies of FePt nanoparticles for dual modal CT/MRI molecular imaging. J. Am. Chem. Soc. 132(38), 13270-13278 (2010).

105 Granade TC, Workman S, Wells SK, Holder AN, Owen SM, Pau CP. Rapid detection and differentiation of antibodies to HIV-1 and HIV-2 using multivalent antigens and magnetic immunochromatography testing. Clin. Vaccine Immunol. 17(6), 1034-1039 (2010).

106 Puertas S, Moros M, Fernández-Pacheco R, Ibarra M, Grazú V, De La Fuente J. Designing novel nano-immunoassays: antibody orientation versus sensitivity. J. Phys. D. Appl. Phys. 43(47), 474012 (2010).

107 Puertas S, Batalla P, Moros M et al. Taking advantage of unspecific interactions to produce highly active magnetic nanoparticle-antibody conjugates. ACS Nano 5(6), 4521-4528 (2011).

108 Liu C, Gao Z, Zeng J et al. Magnetic/upconversion fluorescent NaGdF4:Yb,Er nanoparticle-based dual-modal molecular probes for imaging tiny tumors in vivo. ACS Nano 7(8), 7227-7240 (2013).

109 Guo Q, Liu Y, Xu K, Ren K, Sun W. Mouse lymphatic endothelial cell targeted probes: anti-LYVE-1 antibody-based magnetic nanoparticles. Int. J. Nanomed. 8, 2273-2284 (2013).

110 Zhou J, Leuschner C, Kumar C, Hormes JF, Soboyejo WO. Sub-cellular accumulation of magnetic nanoparticles in breast tumors and metastases. Biomaterials 27(9), 2001-2008 (2006).

111 Vlahov IR, Leamon CP. Engineering folate-drug conjugates to target cancer: from chemistry to clinic. Bioconjug. Chem. 23(7), 1357-1369 (2012). 
112 Sonvico F, Mornet S, Vasseur S et al. Folate-conjugated iron oxide nanoparticles for solid tumor targeting as potential specific magnetic hyperthermia mediators: synthesis, physicochemical characterization, and in vitro experiments. Bioconjug. Chem. 16(5), 1181-1188 (2005).

113 Zhang L, Gong F, Zhang F, Ma J, Zhang P, Shen J. Targeted therapy for human hepatic carcinoma cells using folate-functionalized polymeric micelles loaded with superparamagnetic iron oxide and sorafenib in vitro. Int. J. Nanomed. 8, 1517-1524 (2013).

114 Li D, Zhang YT, Yu M, Guo J, Chaudhary D, Wang CC. Cancer therapy and fluorescence imaging using the active release of doxorubicin from MSPs/Ni-LDH folate targeting nanoparticles. Biomaterials 34(32), 7913-7922 (2013).

115 Sahoo B, Devi KS, Banerjee R, Maiti TK, Pramanik P, Dhara D. Thermal and $\mathrm{pH}$ responsive polymer-tethered multifunctional magnetic nanoparticles for targeted delivery of anticancer drug. ACS Appl. Mater. Int. 5(9), 3884-3893 (2013).

116 Zhang C, Jugold M, Woenne EC et al. Specific targeting of tumor angiogenesis by RGD-conjugated ultrasmall superparamagnetic iron oxide particles using a clinical 1.5-T magnetic resonance scanner. Cancer Res. 67(4), 1555-1562 (2007).

117 Zheng S, Huang M, Hong R et al. RGD-conjugated iron oxide magnetic nanoparticles for magnetic resonance imaging contrast enhancement and hyperthermia. J. Biomater. Appl. doi:10.1177/0885328213493486 (2013) (Epub ahead of print).

118 Kievit FM, Veiseh O, Fang C et al. Chlorotoxin labeled magnetic nanovectors for targeted gene delivery to glioma. ACS Nano 4(8), 4587-4594 (2010).

119 Essinger-Hileman E, Popczun E, Schaak R. Magnetic separation of colloidal nanoparticle mixtures using a material specific peptide. Chem. Commun. 49(48), 5471-5473 (2013).

120 Tresilwised N, Pithayanukul P, Holm PS, Schillinger U, Plank C, Mykhaylyk O. Effects of nanoparticle coatings on the activity of oncolytic adenovirus-magnetic nanoparticle complexes. Biomaterials 33(1), 256-269 (2012).

121 Anton M, Wolf A, Mykhaylyk O, Koch C, Gansbacher B, Plank C. Optimizing adenoviral transduction of endothelial cells under flow conditions. Pharmaceut. Res. 29(5), 1219-1231 (2012).

122 Chorny M, Fishbein I, Tengood JE, Adamo RF, Alferiev IS, Levy RJ. Site-specific gene delivery to stented arteries using magnetically guided zinc oleate-based nanoparticles loaded with adenoviral vectors. FASEB J. 27(6), 2198-2206 (2013).

123 Zhang Y, Li W, Ou L et al. Targeted delivery of human VEGF gene via complexes of magnetic nanoparticleadenoviral vectors enhanced cardiac regeneration. PLoS ONE 7(7), e39490 (2012).

124 Shamim N, Hong L, Hidajat K, Uddin MS. Thermosensitive-polymer-coated magnetic nanoparticles: adsorption and desorption of bovine serum albumin. J. Coll. Int. Sci. 304(1), 1-8 (2006).

125 Wang C, Qiao L, Zhang Q, Yan H, Liu K. Enhanced cell uptake of superparamagnetic iron oxide nanoparticles through direct chemisorption of FITC-Tat-PEG(6)(0) (0)-b-poly (glycerol monoacrylate). Int. J. Pharmaceut. 430(1-2), 372-380 (2012).

126 Jiang W, Xie H, Ghoorah D et al. Conjugation of functionalized SPIONs with transferrin for targeting and imaging brain glial tumors in rat model. PLoS ONE 7(5), e37376 (2012).

127 Di Marco M, Port M, Couvreur P, Dubernet C, Ballirano P, Sadun C. Structural characterization of ultrasmall superparamagnetic iron oxide (USPIO) particles in aqueous suspension by energy dispersive x-ray diffraction (EDXD). J. Am. Chem. Soc. 128(31), 10054-10059 (2006).

128 Choi HS, Liu W, Misra P et al. Renal clearance of quantum dots. Nat. Biotechnol. 25(10), 1165-1170 (2007).

- Good review of clearance mechanisms for nanomedicines.

129 Fadeel B, Garcia-Bennett AE. Better safe than sorry: understanding the toxicological properties of inorganic nanoparticles manufactured for biomedical applications. Adv. Drug Deliv. Rev. 62(3), 362-374 (2010).

- A descriptive review of the toxicological profiles of inorganic nanoparticulates including MNPs.

130 Mahmoudi M, Sant S, Wang B, Laurent S, Sen T. Superparamagnetic iron oxide nanoparticles (SPIONs): development, surface modification and applications in chemotherapy. Adv. Drug Deliv. Rev. 63(1-2), 24-46 (2011).

131 Acharya S, Sahoo SK. PLGA nanoparticles containing various anticancer agents and tumour delivery by EPR effect. Adv. Drug Deliv. Rev. 63(3), 170-183 (2011).

132 Huang X, Li L, Liu T et al. The shape effect of mesoporous silica nanoparticles on biodistribution, clearance, and biocompatibility in vivo. ACS Nano 5(7), 5390-5399 (2011).

133 Alexis F, Pridgen E, Molnar LK, Farokhzad OC. Factors affecting the clearance and biodistribution of polymeric nanoparticles. Mol. Pharmaceut. 5(4), 505-515 (2008).

- Detailed review of the physicochemical and biological properties of nanoparticlulate surface modifications that help avoid nonspecific reticuloendothelial system clearance.

134 Osaka T, Nakanishi T, Shanmugam S, Takahama S, Zhang H. Effect of surface charge of magnetite nanoparticles on their internalization into breast cancer and umbilical vein endothelial cells. Colloids Surf. B Biointerface 71(2), 325-330 (2009).

135 Moghimi SM, Hunter AC, Murray JC. Long-circulating and target-specific nanoparticles: theory to practice. Pharmacol. Rev. 53(2), 283-318 (2001).

136 Duran JD, Arias JL, Gallardo V, Delgado AV. Magnetic colloids as drug vehicles. J. Pharm. Sci. 97(8), 2948-2983 (2008).

137 Qi J, Lu Y, Wu W. Absorption, disposition and pharmacokinetics of solid lipid nanoparticles. Curr. Drug Metab. 13(4), 418-428 (2012).

138 Thode K, Muller RH, Kresse M. Two-time window and multiangle photon correlation spectroscopy size and zeta potential analysis-highly sensitive rapid assay for dispersion stability. J. Pharm. Sci. 89(10), 1317-1324 (2000).

139 Phenrat T, Saleh N, Sirk K, Tilton RD, Lowry GV. Aggregation and sedimentation of aqueous nanoscale 
zerovalent iron dispersions. Environ. Sci. Technol. 41(1), 284-290 (2007).

140 Amstad E, Gillich T, Bilecka I, Textor M, Reimhult E. Ultrastable iron oxide nanoparticle colloidal suspensions using dispersants with catechol-derived anchor groups. Nano Lett. 9(12), 4042-4048 (2009).

141 Viota JL, de Vicente J, Duran JD, Delgado AV. Stabilization of magnetorheological suspensions by polyacrylic acid polymers. J. Colloid Int. Sci. 284(2), 527-541 (2005).

142 Wang C, Xu H, Liang C et al. Iron oxide @ polypyrrole nanoparticles as a multifunctional drug carrier for remotely controlled cancer therapy with synergistic antitumor effect. ACS Nano 7(8), 6782-6795 (2013).

143 Nair M, Guduru R, Liang P, Hong J, Sagar V, Khizroev $S$. Externally controlled on-demand release of anti-HIV drug using magneto-electric nanoparticles as carriers. Nat. Commun. 4, 1707 (2013).

144 Shen JM, Yin T, Tian XZ, Gao FY, Xu S. Surface chargeswitchable polymeric magnetic nanoparticles for the controlled release of anticancer drug. ACS Appl. Mat. Inter. 5(15), 7014-7024 (2013).

145 Mazur M, Barras A, Kuncser V et al. Iron oxide magnetic nanoparticles with versatile surface functions based on dopamine anchors. Nanoscale 5(7), 2692-2702 (2013).

146 Wahajuddin, Arora S. Superparamagnetic iron oxide nanoparticles: magnetic nanoplatforms as drug carriers. Int. J. Nanomed. 7, 3445-3471 (2012).

$147 \mathrm{Hu}$ SH, Hsieh TY, Chiang CS et al. Surfactant-free, lipopolymersomes stabilized by Iron oxide nanoparticles/polymer interlayer for synergistically targeted and magnetically guided gene delivery. Adv. Healthcare Mat. doi:10.1002/ adhm.201300122 (2013) (Epub ahead of print).

148 Wei H, Insin N, Lee J et al. Compact zwitterion-coated iron oxide nanoparticles for biological applications. Nano Lett. 12(1), 22-25 (2012).
149 Qu JB, Shao HH, Jing GL, Huang F. PEG-chitosan-coated iron oxide nanoparticles with high saturated magnetization as carriers of 10-hydroxycamptothecin: preparation, characterization and cytotoxicity studies. Colloids Surf. B Biointerface 102, 37-44 (2013).

150 Clares B, Biedma-Ortiz RA, Saez-Fernandez E et al. Nanoengineering of 5-fluorouracil-loaded magnetoliposomes for combined hyperthermia and chemotherapy against colon cancer. Eur. J. Pharm. Biopharm. 85(3 Pt A), 329-338 (2013).

151 García-Jimeno S, Escribano E, Queralt J, Estelrich J. External magnetic field-induced selective biodistribution of magnetoliposomes in mice. Nanoscale Res. Lett. 7(1), 1-7 (2012).

152 Lee N, Hyeon T. Designed synthesis of uniformly sized iron oxide nanoparticles for efficient magnetic resonance imaging contrast agents. Chem. Soc. Rev. 41(7), 2575-2589 (2012).

153 Banerjee R, Katsenovich Y, Lagos L, McIintosh M, Zhang X, Li CZ. Nanomedicine: magnetic nanoparticles and their biomedical applications. Curr. Med. Chem. 17(27), 3120-3141 (2010).

154 Corot C, Robert P, Idee JM, Port M. Recent advances in iron oxide nanocrystal technology for medical imaging. $A d v$. Drug Deliv. Rev. 58(14), 1471-1504 (2006).

155 Lubbe AS, Bergemann C, Riess $\mathrm{H}$ et al. Clinical experiences with magnetic drug targeting: a Phase I study with 4 '-epidoxorubicin in 14 patients with advanced solid tumors. Cancer Res. 56(20), 4686-4693 (1996).

156 Johannsen M, Thiesen B, Wust P, Jordan A. Magnetic nanoparticle hyperthermia for prostate cancer. Int. J. Hypertherm. 26(8), 790-795 (2010).

157 Maier-Hauff K, Ulrich F, Nestler D et al. Efficacy and safety of intratumoral thermotherapy using magnetic iron-oxide nanoparticles combined with external beam radiotherapy on patients with recurrent glioblastoma multiforme. J. Neurooncol. 103(2), 317-324 (2011). 